\title{
Order-Disorder Transition and Critical Micelle Temperature in Concentrated Block Copolymer Solutions
}

\author{
Moon Jeong Park and Kookheon Char* \\ School of Chemical Engineering \& NANO Systems Institute, National Core Research Center (NCRC), \\ Seoul National University, Seoul 151-744, Korea \\ Joona Bang and Timothy P. Lodge* \\ Department of Chemical Engineering \& Materials Science, Department of Chemistry, University of \\ Minnesota, Minneapolis, Minnesota 55455
}

Received November 9, 2004; Revised Manuscript Received December 30, 2004

\begin{abstract}
The phase behavior of symmetric styrene-isoprene (SI) diblock copolymers in selective solvents in the vicinity of the order-disorder transition (ODT) was investigated by small-angle neutron scattering (SANS). Particular emphasis is placed on the region just above the ODT, where a disordered phase of micelles is observed. To understand morphological changes in more detail, one of the blocks is deuterated, i.e., PS $-d$ PI and $d$ PS-PI, and the scattering length density (SLD) of the solvents used were identical to the SLD of the corona chains. Two approaches for the analysis of SANS data were taken: the generalized indirect Fourier transformation (GIFT) and direct model fitting with relevant form and structure factors. With increasing temperature, the micellar aggregation number decreases, and the core radius is roughly maintained by the increased solvent swelling of the core. The critical micelle temperature (cmt) is experimentally described in the present study by an abrupt decrease in the size, aggregation number, and volume fraction of micelles. The micelles were found to dissociate into free chains approximately $20-30{ }^{\circ} \mathrm{C}$ higher than the $T_{\mathrm{ODT}}$, and the $\mathrm{cmt}$ is found to be quite close to the mean-field spinodal temperature $T_{\mathrm{S}}$. A mean-field temperature, $T_{\mathrm{MF}}$, is located by the crossover temperature from the linear behavior of $I_{\max }{ }^{-1}$ vs $T^{-1}$ plot, and the structure factor above the experimentally determined $T_{\mathrm{MF}}$ is also found to be in good agreement with the Leibler-Landau-type mean-field theory. We examined two different ODTs: body-centered cubic lattices to disorder and hexagonal cylinders to disorder, with similar results in both cases.
\end{abstract}

\section{Introduction}

Numerous studies have been devoted to the fascinating phase transitions exhibited in block copolymers. ${ }^{1-10}$ It is now well established both theoretically and experimentally for bulk diblock copolymers how the phase transitions (i.e., order-order and order-disorder transitions) depend on block composition and temperature. In particular, although the mean-field theory of Leibler ${ }^{1}$ anticipates a first-order phase transition between a disordered melt and a bcc lattice of spherical micelles for any composition except for perfect symmetry, experimentally one finds the order-disorder transition to connect the disordered state directly to lamellae, hexagonal cylinders, double gyroid, or bcc spheres, depending on composition. Fredrickson and Helfand ${ }^{3}$ argued that block copolymers fall in the Brazovskii universality class and that substantial spatial fluctuations in composition could stabilize the disordered phase, thereby accounting for the direct access to the various ordered states. Extensive experimental evidence of such fluctuations has been documented, and a variety of other theoretical approaches have also been brought to bear. ${ }^{8-22}$ However, it is fair to say that a complete picture of the disordered state in the proximity of the ODT is still lacking.

One particularly interesting aspect of this regime arises for asymmetric copolymers, which adopt the bcc phase below the ODT. ${ }^{9,10,23-30}$ Several authors observed

* Authors for correspondence: (K.C.) khchar@plaza.snu.ac.kr; (T.P.L) lodge@chem.umn.edu. that the disordered phase just above the ODT exhibited evidence of spherical micelles in a liquidlike state. The resulting ODT therefore reflects the loss of long-range order and identifiable symmetry, but does not result in complete homogenization of the blocks. Some authors have suggested distinguishing this ODT from that observed for gyroid, cylinder, or lamellar samples, by terming it the lattice disordering transition. ${ }^{27-30}$ If there are distinct micelles present above the ODT, then at sufficiently high-temperature such micelles should disperse in favor of free chains. Evidence for a "critical micelle temperature" (cmt) has been presented in bulk diblocks, although it should be recognized that such a cmt does not represent a true thermodynamic phase transition. ${ }^{26,30-33}$ It is of interest, therefore, to see whether such a "disordered micelle state" and an associated cmt can be observed in block copolymer solutions above the ODT.

Recently, Semenov's strong-segregation theory ${ }^{34}$ for highly asymmetric block copolymers was extended to consider the possible stability of a disordered micelle regime above the ODT. ${ }^{26}$ The existence of disordered micelles was indeed confirmed in sphere-forming diblock copolymers at elevated temperatures, ${ }^{28-30}$ in contrast to classical mean-field calculations. 1,35,36 Experimentally, ${ }^{31}$ detailed analysis of SANS data in the bulk suggests that the micelles remain relatively unchanged as the temperature is increased, but their number density and volume fraction decrease steadily.

Although considerable attention has already been paid to micellization in dilute solutions, there have been fewer reports on concentrated block copolymer solutions. 
Pioneering studies on block copolymer solutions were performed by Hashimoto and co-workers. ${ }^{37,38}$ For asymmetric polystyrene-polybutadiene (PS-PB) block copolymers in the $\mathrm{PB}$-selective solvent tetradecane (C14), Hashimoto and co-workers found that at a fixed concentration, increasing temperature leads to a loss of long-range order, signaling a melting from an ordered lattice to a liquidlike micellar solution; at even higher temperatures, the micelles eventually dissolve into a homogeneous solution.

Hamley and co-workers ${ }^{39}$ investigated the phase behavior of asymmetric PS-PI and PS-PI-PS block copolymers in the PS-selective solvent di-n-butylphthalate (DBP). The hexagonal cylinder (hex) and lamellar microstructures in the concentration range $0 \leq \phi \leq 0.4$ dissolve into micellar solutions at $T_{\mathrm{ODT}}$. Above $T_{\mathrm{ODT}}$ there is a significant range of temperature over which the micellar interactions persist before the micelles are fully dissociated. On the basis of a combination of SAXS and rheology measurements, they proposed an "approximate phase diagram" where the micelles were dissociated into free chains approximately $30^{\circ} \mathrm{C}$ above the $T_{\text {ODT. }}$. The ODT temperature in concentrated block copolymer solutions was found to be essentially independent of concentration and it is also interesting to note that the ODTs in concentrated solutions coincided with the cmts in dilute solutions, indicating that the ODT is dictated by the PI-solvent interaction, rather than the PI-PS block interaction. ${ }^{40,41}$

The work by Hamley and co-workers ${ }^{39}$ raises two fundamental issues: (1) What would the effect of the addition of a selective solvent on the ODT of symmetric diblock copolymers be? (2) Is there any difference in structure and other characteristics of disordered micelles caused by different ordered microstructures, i.e., body-centered cubic (bcc) and hex, prior to the disordered state?

In this study, we examine nondilute block copolymer solutions, focusing on a symmetric diblock copolymer in selective solvents. To investigate the structural features in the disordered micelle regime, a detailed experimental examination of two ODTs is presented: from bcc spheres and from hexagonal cylinders. To clarify two structural aspect of block copolymer solutions with SANS, one of the blocks is deuterated and solvents selective to the protonated block were used, i.e., PS- $b$ $d \mathrm{PI}$ in dialkyl phthalates and $d \mathrm{PS}-b$-PI in tetradecane. The ODT is defined in the present study as the transition temperature from the bcc or hex phase to disordered micelles and the cmt as the temperature for the dissolution of micelles. A qualitative criterion for the $\mathrm{cmt}$ is also suggested by the SANS data. In a related study, the detailed micellar characteristics of the same copolymers were studied by SANS in dilute solution, as a function of temperature up to the cmt. ${ }^{42}$

\section{Experimental Section}

Materials. Two nearly symmetric diblock copolymers were synthesized by a standard anionic polymerization procedure..$^{40}$ One copolymer, designated $\mathrm{S} d \mathrm{I}(15-14)$, incorporated protonated styrene and perdeuterated isoprene, while the other, designated $d \mathrm{SI}(16-15)$, had perdeuterated styrene and protonated isoprene. The deuterated monomers were purchased from Polymer Source, Inc. Styrene (protonated or perdeuterated) was purified by stirring over calcium hydride for $12 \mathrm{~h}$, followed by vacuum distillation with $n$-butyllithium for $6 \mathrm{~h}$. Isoprene (protonated or perdeuterated) was treated with dibutylmagnesium for $3 \mathrm{~h}$, followed by $n$-butyllithium for $6 \mathrm{~h}$.
Table 1. Scattering Length Densities (SLDs) of Materials Used in Present Study

\begin{tabular}{llrcc}
\hline species & formula & $\begin{array}{r}\text { mol wt } \\
(\mathrm{g} / \mathrm{mol})\end{array}$ & $\begin{array}{c}\text { density } \\
\left(\mathrm{g} / \mathrm{cm}^{3}\right)\end{array}$ & $\begin{array}{c}\text { scattering length } \\
\text { density } \\
\left(10^{-6} \AA^{-2}\right)\end{array}$ \\
\hline $\mathrm{PS}$ & $\mathrm{C}_{8} \mathrm{H}_{8}$ & 104.15 & 1.047 & 1.41 \\
$d \mathrm{PS}$ & $\mathrm{C}_{8} \mathrm{D}_{8}$ & 112.22 & 1.128 & 6.45 \\
$\mathrm{PI}$ & $\mathrm{C}_{5} \mathrm{H}_{8}$ & 68.12 & 0.913 & 0.27 \\
$d \mathrm{PI}$ & $\mathrm{C}_{5} \mathrm{D}_{8}$ & 76.09 & 0.986 & 6.75 \\
$\mathrm{DEP}$ & $\mathrm{C}_{12} \mathrm{H}_{14} \mathrm{O}_{4}$ & 232.16 & 1.118 & 1.53 \\
$\mathrm{DBP}$ & $\mathrm{C}_{16} \mathrm{H}_{22} \mathrm{O}_{4}$ & 278.35 & 1.043 & 1.07 \\
$\mathrm{C} 14$ & $\mathrm{C}_{14} \mathrm{H}_{30}$ & 198.40 & 0.763 & -0.44 \\
$d \mathrm{C} 14$ & $\mathrm{C}_{14} \mathrm{D}_{30}$ & 228.66 & 0.882 & 6.81
\end{tabular}

Cyclohexane was used as the polymerization solvent and was distilled from $n$-butyllithium. Using sec-butyllithium as an initiator, the styrene was polymerized for $4 \mathrm{~h}$ at $45^{\circ} \mathrm{C}$, followed by the addition of isoprene and polymerization for $4 \mathrm{~h}$ at the same temperature.

The polymers were characterized by size exclusion chromatography (SEC) equipped with both refractive index and multiangle light scattering detectors (Wyatt Optilab and Dawn), and by ${ }^{1} \mathrm{H}$ NMR spectroscopy. A small amount of the styrene block was sampled before adding the isoprene monomer and SEC yielded number-average molecular weights of $14100(\mathrm{PS})$ and 15400 ( $d \mathrm{PS}$ ) with polydispersity indices around 1.02. After growing the isoprene block, a light scattering instrument was incorporated in the SEC apparatus to determine the absolute molecular weight. ${ }^{43}$ The refractive index increment was determined with a differential refractometer independently, and this value was then used in the analysis of the chromatogram. ${ }^{44}$ The resulting block molecular weights were $15400(\mathrm{~S})$ and $14100(d \mathrm{I}) \mathrm{g} / \mathrm{mol}$ for $\mathrm{S} d \mathrm{I}(15-14)$ and 15800 $(d \mathrm{~S})$ and $15400(\mathrm{I}) \mathrm{g} / \mathrm{mol}$ for $d \mathrm{SI}(16-15)$, respectively. The polydispersity indices for both $\mathrm{S} d \mathrm{I}(15-14)$ and $d \mathrm{SI}(16-15)$ are 1.04. ${ }^{1} \mathrm{H}$ NMR spectroscopy (Varian VXR-500 MHz) was used to determine the composition and to estimate the mole percent of 1,4-addition of the PI block $(94 \pm 1 \%)$. Two PS-selective solvents, diethyl phthalate (DEP) and di- $n$-butyl phthalate (DBP), and one PI-selective solvent, $n$-tetradecane (C14), were obtained from Aldrich. Perdeuterated tetradecane $(d-C 14)$ was obtained from $\mathrm{C} / \mathrm{D} / \mathrm{N}$ Isotope Inc.

Solutions of $\mathrm{S} d \mathrm{I}(15-14)$ in a mixture of $75: 25$ vol \% DEP: $\mathrm{DBP}$ and $d \mathrm{SI}(16-15)$ in $\mathrm{C} 14$ were prepared with polymer concentrations of 25 and $40 \mathrm{vol} \%$ using $\mathrm{CH}_{2} \mathrm{Cl}_{2}$ as a cosolvent; the $\mathrm{CH}_{2} \mathrm{Cl}_{2}$ was later removed under a gentle flow of nitrogen until the solution reached a constant weight. The $25 \%$ and $40 \%$ solutions were intentionally chosen to access the bcc/disorder and hex/disorder transitions, respectively, as a function of temperature. ${ }^{40,41,45}$

Small-Angle Neutron Scattering (SANS). SANS measurements were performed using the $30 \mathrm{~m}$ NG7 beamline at the National Institute of Standards and Technology (NIST). A sample-to-detector distance of $7.05 \mathrm{~m}$ and a neutron wavelength $(\lambda)$ of $6 \AA$ with $\Delta \lambda / \lambda=0.10$ were used to access scattering vectors $q(q=4 \pi \sin (\theta / 2) / \lambda$, where $\theta$ is the scattering angle) in the range $0.064-0.975 \mathrm{~nm}^{-1}$. A heated sample chamber was employed for temperature control, with an accuracy of $\pm 0.1{ }^{\circ} \mathrm{C}$. The typical exposure time was $5 \mathrm{~min}$ at a given temperature and each solution was annealed for at least $10 \mathrm{~min}$ at a given temperature before measurement. For $\mathrm{S} d \mathrm{I}(15-14)$, a mixture of PS-selective solvents DEP and DBP with a volume ratio of 3 to 1 , was chosen for the contrast matching of the scattering length density (SLD) with the PS coronas in the micelles, thereby enabling detailed examination of the $d$ PI cores. The contrast matching method was also applied to $d \mathrm{SI}(16-15)$ by using a mixture of tetradecane (C14) and deuterated tetradecane $(d \mathrm{C} 14)$ with a volume ratio of 9 to 1 . Since C14 is selective for the PI block, the $d \mathrm{PS}$ core dominates the SANS. The solutions were placed between two sealed quartz windows with a flight path of ca. $1 \mathrm{~mm}$. SLDs and molecular characteristics of materials used for the SANS experiments are summarized in Table 1. 


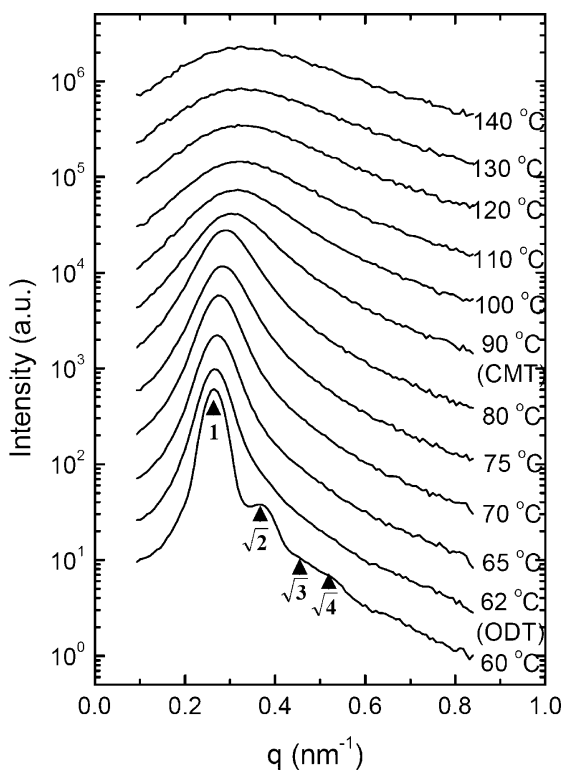

Figure 1. SANS profiles for a $25 \%$ SdI(15-14) in DEP/DBP $(75 / 25 \mathrm{vol} \%)$ solution as a function of temperature. The filled triangles for the SANS profile at $60{ }^{\circ} \mathrm{C}$ indicate the Bragg reflections corresponding to the bcc phase. Each profile is shifted vertically by half a decade relative to the profile for immediately previous temperature for clarity. $T_{\mathrm{ODT}}$ is located at $62{ }^{\circ} \mathrm{C}$ and we also observed an abrupt decrease in the peak intensity at around $90{ }^{\circ} \mathrm{C}$, which is attributed to the cmt.

\section{Results and Discussion}

ODT from Bcc Microstructure. Figure 1 presents the SANS profiles (scattering intensity $I(q)$ against scattering vector q) for the $25 \% \mathrm{~S} d \mathrm{I}(15-14)$ in $\mathrm{DEP} /$ DBP measured at different temperatures to show the behavior at and above the ODT in more detail. Each scattering profile was shifted vertically for clarity. The scattering intensity decreases significantly with increasing temperature indicating the classical UCODT-type phase behavior. Below $60^{\circ} \mathrm{C}$, the higher order peaks at $\sqrt{2} q_{\max }, \sqrt{3} q_{\max }$, and $\sqrt{4} q_{\max }$ indicate the microphaseseparated bcc structure, which completely disappear at a temperature between 60 and $62{ }^{\circ} \mathrm{C}$. Consequently, the $T_{\text {ODT }}$ is considered to be located between 60 and $62{ }^{\circ} \mathrm{C}$. Around $90{ }^{\circ} \mathrm{C}$, there is an additional abrupt decrease in the peak intensity and increase in peak width, which we attribute to the $\mathrm{cmt}$ at which the micelle-to-free chain equilibrium is shifted toward the free chains. Upon further heating, the main peak intensity originating from the intermicellar interaction becomes negligible, with only the broad correlation hole contribution remaining, indicating a fully disordered copolymer solution.

To facilitate the comparison of experimental data obtained here with theory, the inverse peak intensity, $I_{\max }{ }^{-1}$, the square of the full-width at half-maximum (fwhm) of the main peak, $\sigma_{q}^{2}$, and the characteristic length $D$ defined by $D=2 \pi / q_{\max }$ with $q_{\max }$ being the scattering vector at the first peak position, are plotted against the inverse of absolute temperature, $T^{-1}$, as shown in Figure 2. The first-order transition at $T_{\mathrm{ODT}}=$ $62{ }^{\circ} \mathrm{C}$ is confirmed in the insets via discontinuous changes in both $I_{\max }{ }^{-1}$ and $\sigma_{q}{ }^{2}$. In the disordered state of block copolymers, the mean-field theory ${ }^{1}$ for a UCODTtype block copolymer predicts that both $I_{\max }{ }^{-1}$ and $\sigma_{q}{ }^{2}$ are proportional to $T^{-1}$. As shown in Figure 2, both $I_{\max }{ }^{-1}$ and $\sigma_{q}{ }^{2}$ exhibit a fairly wide region of linear

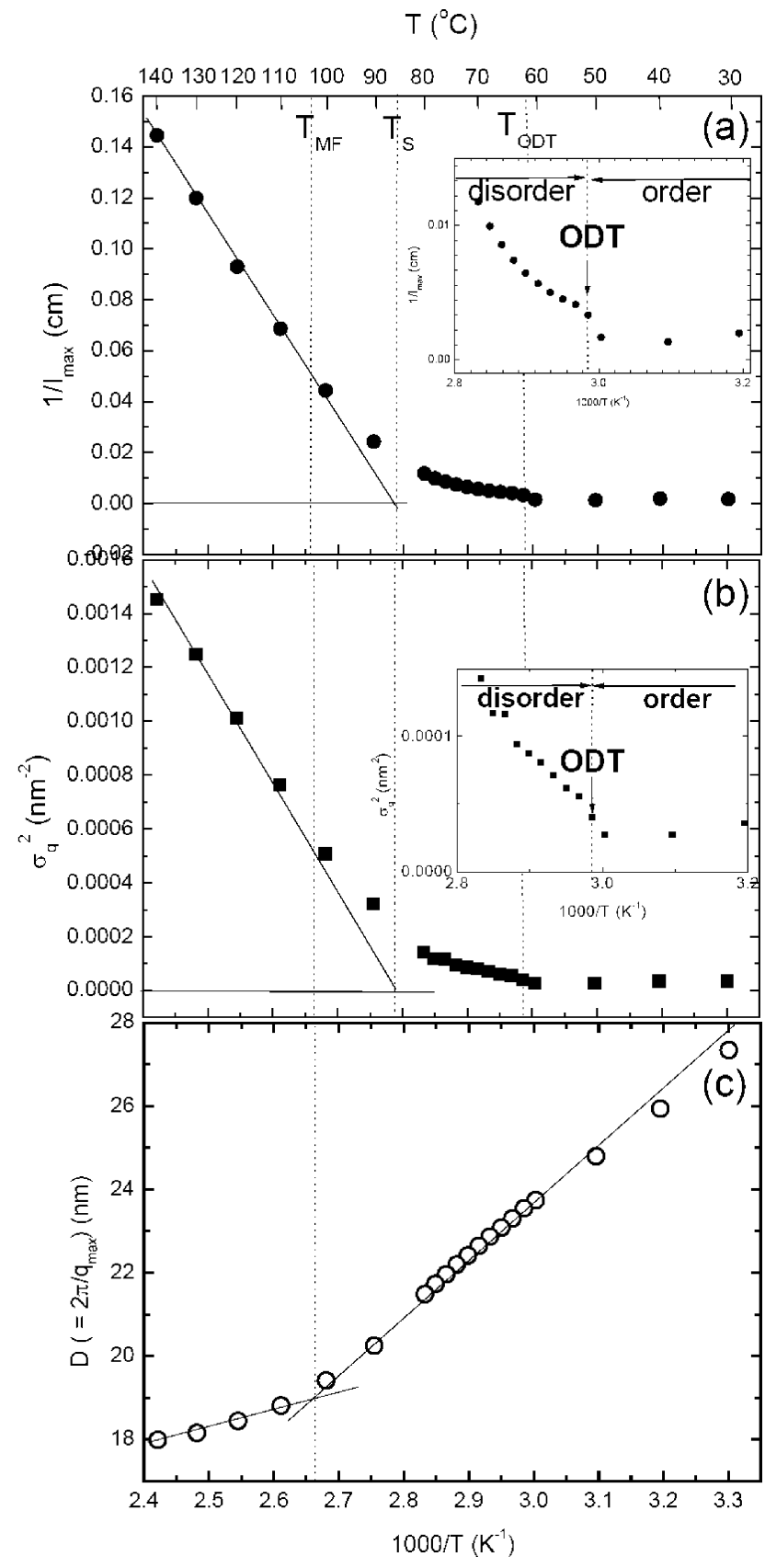

Figure 2. Temperature dependence of (a) $I_{\max }{ }^{-1}$, (b) $\sigma_{q}{ }^{2}$, and (c) $D$ for a $25 \% \mathrm{~S} d \mathrm{I}(15-14)$ in $\mathrm{DEP} / \mathrm{DBP}(75 / 25$ vol \%) solution. The lines in parts $\mathrm{a}$ and $\mathrm{b}$ are fitted to the data as predicted from the mean-field theory. Insets in parts a and $b$ highlight the discontinuous change in both quantities near the ODT. The positions of mean-field temperature $\left(T_{\mathrm{MF}}\right)$, spinodal temperature $\left(T_{\mathrm{S}}\right)$, and order-disorder transition temperature $\left(T_{\mathrm{ODT}}\right)$ are also indicated in the figure.

behavior with respect to $T^{-1}$. The thermal fluctuation effect near the ODT is known to cause a deviation from the linear relationship and the crossover temperature from the mean-field to the thermal fluctuation regime in the disordered state was defined as $T_{\mathrm{MF}}$. We also note in Figure 2 that there is a clear-cut crossover of $D$ upon heating the sample through the mean-field temperature, and $T_{\mathrm{MF}}=104^{\circ} \mathrm{C}$ was thus determined. The mean-field spinodal temperature, $T_{\mathrm{S}}=86^{\circ} \mathrm{C}$, is defined by the temperature at which both $I_{\max }{ }^{-1}$ and $\sigma_{q}^{2}$ become zero by the linear extrapolation from the mean-field region. It is intriguing to note that the mean-field spinodal 
temperature $T_{\mathrm{S}}$ is quite close to the cmt as defined in Figure 1.

We have taken two approaches for the analysis of the SANS data to investigate the disordered micelle regime in more detail. One employs the model-independent generalized indirect Fourier transformation (GIFT) developed by Glatter and co-workers ${ }^{46}$ and the other uses direct model fitting with relevant form and structure factors. The GIFT method separates the form factor $P(q)$ (i.e., intraparticle scattering) and the structure factor $S(q)$ (i.e., interparticle scattering) contributions. In the case of a uniform dispersion of monodisperse, homogeneous, and isotropic spherical particles, no orientation effect is taken into account so that the total scattering intensity $I(q)$ can be written

$$
I(q)=N P(q) \mathrm{S}(q)
$$

where $N$ is the number of particles. From the form factor $P(q)$, it is possible to obtain the pair distance distribution function, $p(r)$, by the spatially averaged Fourier transformation:

$$
P(q)=4 \pi \int_{0}^{\infty} p(r) \frac{\sin (q r)}{q r} \mathrm{~d} r
$$

In real $(r)$ space, the interparticle interactions are described by the total correlation function $h(r)=g(r)-$ 1 with $g(r)$ being the radial distribution function and $r$ the distance between the centers of two particles. ${ }^{47}$ Using the relationship between functions in $q$ and $r$ space, $S(q)$ and $h(r)$, similar to the pair of $P(q)$ and $p(r)$, form another Fourier transform pair:

$$
S(q)=1+4 \pi n \int_{0}^{\infty} h(r) r^{2} \frac{\sin (q r)}{q r} \mathrm{~d} r
$$

Equation 1 applies to the case of an isotropic dispersion of monodisperse spherical particles. However, it has been shown with many examples that by introducing the "averaged structure factor" $S^{\text {ave }}(q)$, with the average taken over the weighted contributions of partial structure factors for individual monodisperse systems. More exactly the "effective structure factor" $S^{e f f}(q)$ can produce improved experimental data fit for polydisperse spheres or cylinders ${ }^{48}$ and even for inhomogeneous particles. ${ }^{46,49}$ In this case $S^{\text {eff }}(q)$ is no longer a single function of the particle distribution in space, but depends on the form amplitudes of the particles. As a result, the structure factor is replaced by the effective structure factor ${ }^{49}$

$$
I(q)=N \overline{P(q)} S^{e f f}(q)
$$

where $\overline{P(q)}$ is the appropriately averaged form factor. The structure factor $S(q)$ is determined by means of a nonlinear least-squares method, and a Lagrange multiplier is systematically varied until the optimum solution is obtained. The $S(q)$ involves up to four adjustable parameters for a given interaction model. We find that the Percus-Yevick (PY) average hard-sphere model, combined with an unconstrained form factor for a polydisperse system, fits the data quite well at temperatures above the ODT.

Figure 3a shows typical pair distance distribution functions, $p(r)$, at different temperatures for the $25 \%$ solution and the decrease in the $d$ PI core size with the increase in temperature is clearly visible. The resulting number-average micelle core radii, $R_{c}$, extracted from
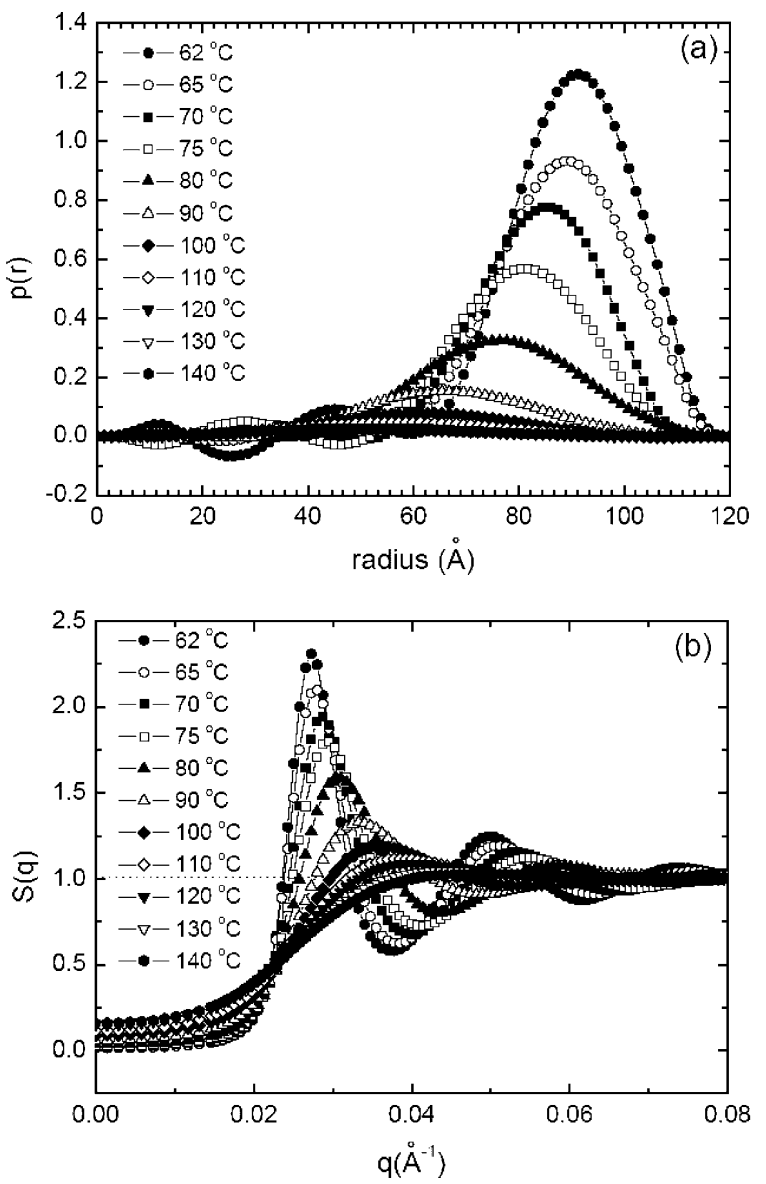

Figure 3. Representative (a) pair distance distribution functions, $p(r)$, and (b) structure factors, $S(q)$, obtained from the GIFT at indicated temperatures for a $25 \% \mathrm{~S} d \mathrm{I}(15-14)$ in DEP/ DBP $(75 / 25$ vol \%).

Table 2. Summary of GIFT Fitting Parameters for a 25\% SdI $(15-14)$ in DEP/DBP (75/25 vol \%)

\begin{tabular}{rrrcrc}
\hline$T,{ }^{\circ} \mathrm{C}$ & $R_{\mathrm{c}}, \AA$ & $R_{\mathrm{hs}}, \AA$ & $R_{\mathrm{hs}}-R_{\mathrm{c}}, \AA$ & $N_{\mathrm{agg}}$ & $\phi_{\mathrm{hs}}$ \\
\hline 62 & 91.4 & 129.9 & 38.5 & 135 & 0.510 \\
65 & 88.6 & 125.6 & 37.0 & 123 & 0.494 \\
70 & 85.9 & 121.6 & 35.7 & 112 & 0.474 \\
75 & 81.0 & 115.2 & 34.2 & 94 & 0.441 \\
80 & 76.4 & 110.6 & 34.2 & 79 & 0.415 \\
90 & 65.5 & 100.1 & 34.6 & 49 & 0.325 \\
100 & 61.4 & 92.6 & 31.2 & 41 & 0.308 \\
110 & 57.3 & 86.8 & 29.5 & 33 & 0.284 \\
120 & 50.5 & 83.4 & 32.9 & 23 & 0.243 \\
130 & 50.5 & 80.5 & 30 & 23 & 0.241 \\
140 & 49.1 & 79.2 & 30.1 & 21 & 0.236
\end{tabular}

$p(r)$ are listed in Table 2. Since the shape of $p(r)$ is found to be sensitive to the upper bound on $R_{\max }, R_{\max }$ was carefully varied to obtain the best fit. There are small oscillations for $R_{c}<60 \AA$, probably due to the noise in the data at high $q$ and the instrumental smearing. The corresponding structure factors for the $25 \%$ solution at different temperatures obtained from the GIFT analysis are also shown in Figure 3b. The parameters need to determine the structure factor are the effective hard sphere radius, $R_{h s}$, and the volume fraction of effective hard spheres, $\phi_{h s}$. As summarized in Table $2, R_{c}$ and $\phi_{h s}$ decrease steadily for the entire temperature region covered, while the difference between $R_{h s}$ and $R_{c}$ related to the effective thickness of corona remains almost constant with a value between 3.0 and $3.9 \mathrm{~nm}$. The aggregation number of the micelles, assuming no solvent in core, can be estimated from the core size and 
the molecular volume of the $d$ PI block based on the relationship

$$
N_{a g g}=\frac{4 \pi R_{c}^{3}}{3 V_{d P I}}=\frac{4 \pi R_{c}^{3}}{3} \frac{\rho_{d P I} \mathrm{~N}_{\mathrm{A}}}{M_{d P I}}
$$

where $N_{\mathrm{A}}$ is Avogadro's number, $\rho_{\mathrm{dPI}}$ is the density of the $d \mathrm{PI}$ block, and $\mathrm{M}_{d \mathrm{PI}}$ is the molecular weight of the $d$ PI block. At $62{ }^{\circ} \mathrm{C}$, the calculated $N_{\text {agg }}$ is 135 , and this value is in fair agreement with the $N_{\text {agr }}$ estimated directly from the $q_{\max }$ value as follows. At $60^{\circ} \mathrm{C}$, when the solution forms the bcc lattice

$$
\begin{aligned}
& N_{\text {agg }}=\frac{\text { volume per one micelle }}{\text { volume per one chain }}= \\
& \qquad \frac{\phi\left(a^{3} / 2\right) f_{\text {core }}}{\text { molecular volume of core } / N_{A}}
\end{aligned}
$$

where $a$ is the lattice parameter, $a=\sqrt{2} d_{110}=$ $\sqrt{2}\left(2 \pi / q_{\max }\right), \phi$ is the polymer volume fraction, and $f_{\text {core }}$ is a volume fraction of the core block. The $N_{\text {agg }}$ calculated from eq 6 is 152 . This value lies slightly above the value obtained by the fitting with SANS data since the calculation by eq 6 assumes that all chains are in micelles. It is interesting to note that we observed a significant decrease in $\phi_{h s}$ at around $90{ }^{\circ} \mathrm{C}$, which is believed to be the cmt, as mentioned in Figures 1 and 2. Additionally, the oscillations in $S(q)$, indicative of correlations among micelles, almost disappear. This observation implies that the block copolymer chains incorporated into the micelles are dissociated to free chains around this temperature (i.e., $\mathrm{cmt}$ ). The validity of the model fit using eq 4 to scattering profiles above the cmt will be discussed in later.

To confirm the micellar size and the shape, different models of the form factor have been tested to fit the SANS data. A micellar form factor shown in eq 7 consists of the self-correlation of a core, the selfcorrelation of a corona, the cross-terms between the core and the corona, as well as between different corona chains: ${ }^{50,51}$

$$
\begin{gathered}
P_{\text {micelle }}(q)=N \Delta \rho_{\text {core }}{ }^{2} P_{\text {core }}(q)+N \Delta \rho_{\text {corona }}{ }^{2} P_{\text {corona }}(q)+ \\
2 N \Delta \rho_{\text {core }} \Delta \rho_{\text {corona }} S_{\text {core-corona }}(q)+ \\
N(N-1) \Delta \rho_{\text {corona }}{ }^{2} S_{\text {corona-corona }}(q)
\end{gathered}
$$

where $\Delta \rho$ is the contrast of scattering length density (SLD) between core (or corona) and solvent. This expression was also used in our study of the same copolymers in dilute solution. ${ }^{42}$ Since we took advantage of the contrast matching in SLD between the solvent and the corona (i.e., $\Delta \rho_{\text {corona }}=0$ ), the micelle form factor shown in eq 7 can be significantly simplified to

$$
P_{\text {micelle }}(q)=N \Delta \rho_{\text {core }}{ }^{2} \mathrm{P}_{\text {core }}(q)
$$

If we assume a sphere form factor for a spherical micelle with uniform SLD, the micelle form factor can be written as

$$
P_{\text {micelle }}\left(q, R_{c}\right)=\left[\left(\frac{4 \pi}{3} R_{c}^{3}\right) \Delta \rho_{\text {core }} \frac{3 J_{1}\left(q R_{c}\right)}{q R_{c}}\right]^{2}
$$

where $J_{1}(x)\left(J_{1}(x)=(\sin (x)-x \cos (x)) / x^{2}\right)$ is the first-order spherical Bessel function. The polydispersity in $R_{c}$ is taken into account by convoluting the spherical form factor with the Gaussian distribution. Consequently, the $P(q)$ becomes

$$
P\left(q, R_{c}, \sigma_{c}\right)=\int_{0}^{\infty} \exp \left(\frac{-\left(R-R_{c}\right)^{2}}{2 \sigma_{c}{ }^{2}}\right) P_{\text {micelle }}\left(q, R_{c}\right) \mathrm{d} R
$$

where $\sigma_{\mathrm{c}}$ is the width of the distribution.

We also need to take into account the Percus-Yevick (PY) structure factor, $S(q)$, as shown in eq 11 below in order to fit the SANS data, to describe the intermicellar interaction based on hard spheres

$$
S(q)=\frac{1}{1+24 \phi_{h s} \mathrm{G}\left(2 q R_{h s}\right) /\left(2 q R_{h s}\right)}
$$

where $\mathrm{G}$ is a trigonometric function of $A=2 q R_{h s}$ and $\phi_{h s}$ given below:

$$
\begin{aligned}
& G(A)=\left(\frac{\left(1+2 \phi_{h s}\right)^{2}}{\left(1-\phi_{h s}\right)^{4}}\right)\left(\frac{\sin A-A \cos A}{A^{2}}\right)- \\
& \left(\frac{6 \phi_{h s}\left(1+0.5 \phi_{h s}\right)^{2}}{\left(1-\phi_{h s}\right)^{4}}\right)\left(\frac{2 A \sin A+\left(2-A^{2}\right) \cos A-2}{A^{3}}\right)+ \\
& \left(\begin{array}{l}
\left.\frac{0.5 \phi_{h s}\left(1+2 \phi_{h s}\right)^{2}}{\left(1-\phi_{h s}\right)^{4}}\right) \times \\
\left(\left[-A^{4} \cos A+4\left\{\left(3 A^{2}-6\right) \cos A+\left(A^{3}-6 A\right) \sin A+\right.\right.\right. \\
\left.6\}] / A^{5}\right)(12)
\end{array}\right.
\end{aligned}
$$

In the end there are four independent parameters involved in fitting the model-specific scattering intensity: a micelle core radius, $R_{c}$, a polydispersity in micellar core radius, $\sigma_{\mathrm{c}}$, an effective hard-sphere radius, $R_{h s}$, and a volume fraction of effective hard spheres, $\phi_{h s}$.

The calculated neutron scattering intensity is also smeared to fit the measured SANS intensity $I\left(q_{0}\right)$ considering the instrumental resolution function, which can be approximated by the Gaussian function as follows: 52

$$
I\left(q_{0}\right)=\int I(q) \frac{1}{\sqrt{2 \pi} \sigma_{q}} \exp \left(-\frac{1}{2}\left(\frac{q-q_{0}}{\sigma_{q}}\right)^{2}\right) \mathrm{d} q
$$

where $\sigma_{q}$ is determined by the monochromaticity of neutron wavelength, detector resolution, and instrumental geometry. It is, however, noted that in these solutions $\sigma_{q}$ plays a minor role in determining the scattering parameters.

Figure 4 shows the comparison of the SANS data at $70{ }^{\circ} \mathrm{C}$ with the model fits based on both the GIFT and the PY model with a spherical form factor. Both models are in excellent agreement with our experimental data. The resulting parameters obtained from the specific model fits are summarized in Table 3 . Note that the $R_{c}$, $R_{h s}$, and $\phi_{h s}$ values are in close agreement with the values obtained from the GIFT analysis. However, it is quite feasible that a significant amount of solvent can exist within the micelle core near the ODT. If the solvent 


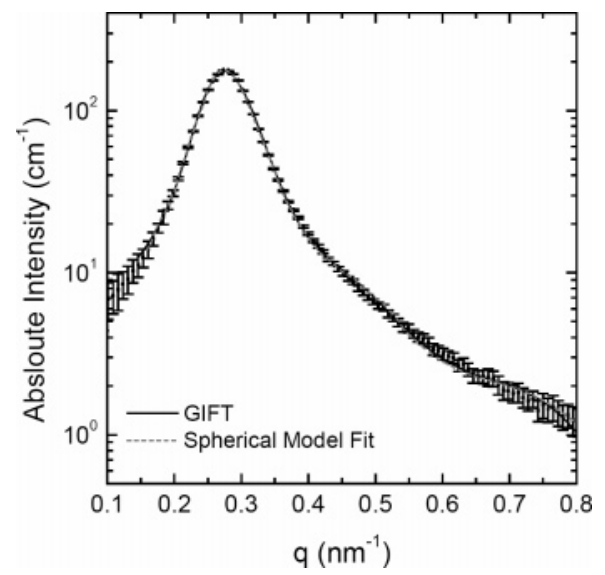

Figure 4. SANS scattering profile for a $25 \% \mathrm{~S} d \mathrm{I}(15-14)$ in DEP/DBP $\left(75 / 25\right.$ vol \%) solution at $62{ }^{\circ} \mathrm{C}$ fitted with both the GIFT and the PY effective hard-sphere model with a spherical form factor

Table 3. Summary of Parameters Obtained from the Percus-Yevick Hard Sphere Model Fits for a 25\% SdI $(15-14)$ in DEP/DBP (75/25 vol \%)

\begin{tabular}{rrrccccc}
\hline$T,{ }^{\circ} \mathrm{C}$ & $R_{\mathrm{c}}, \AA$ & $R_{\mathrm{hs}}, \AA$ & $R_{\mathrm{hs}}-R_{\mathrm{c}}, \AA$ & $N_{\text {agg }}$ & $\phi_{\text {core, sol }}$ & $\sigma_{\mathrm{c}}$ & $\phi_{\mathrm{hs}}$ \\
\hline 62 & 87.1 & 130.0 & 42.9 & 70 & 0.395 & 6.3 & 0.510 \\
65 & 85.2 & 125.5 & 40.3 & 63 & 0.422 & 7.3 & 0.498 \\
70 & 82.3 & 122.0 & 39.7 & 55 & 0.444 & 7.3 & 0.470 \\
75 & 79.0 & 115.5 & 36.5 & 44 & 0.491 & 7.7 & 0.445 \\
80 & 73.6 & 110.0 & 36.4 & 35 & 0.504 & 8.2 & 0.415 \\
90 & 60.8 & 98.3 & 37.5 & 19 & 0.527 & 8.0 & 0.325 \\
100 & 58.4 & 92.6 & 34.2 & 14 & 0.613 & 11.1 & 0.310 \\
110 & 56.1 & 86.5 & 30.4 & 11 & 0.659 & 12.3 & 0.285 \\
120 & 50.5 & 83.1 & 32.6 & 8 & 0.668 & 11.1 & 0.250 \\
130 & 49.6 & 79.2 & 29.6 & 7 & 0.686 & 12.4 & 0.245 \\
140 & 49.1 & 78.5 & 29.4 & 6 & 0.706 & 14.2 & 0.238
\end{tabular}

is allowed to exist within the micelle core ( $d \mathrm{PI}$ in present study), the $\Delta \rho$ in eq 9 is rewritten below:

$$
\begin{array}{r}
\Delta \rho=\left[\frac{3 N_{a g g} V_{d P I}}{4 \pi R_{c}^{3}} \rho_{d P I}+\left(1-\frac{3 N_{a g g} V_{d P I}}{4 \pi R_{c}^{3}}\right) \rho_{S}\right]- \\
\rho_{S}=\frac{3 N_{a g g} V_{d P I}}{4 \pi R_{c}^{3}}\left(\rho_{d P I}-\rho_{S}\right)
\end{array}
$$

The resulting aggregation numbers and volume fractions of solvent in the micelle core are also listed in Table 3. It is interesting to note that the decrease in aggregation number is offset by the increased solvent swelling of the micelle core as the temperature is increased. Above the cmt, the obtained aggregation number is found to be below 20 and the volume fraction of solvent in the micelle core is above $50 \%$, implying that micelles start to break up into free chains at approximately $30{ }^{\circ} \mathrm{C}$ higher than $T_{\text {ODT }}$. Upon further heating, the aggregation number decreases below 10 and the solvent is estimated to exist in the micelle core as much as $70 \%$. It is, however, noted here that the SANS profiles in disordered state, particularly above $T_{\mathrm{MF}}$ should be analyzed by the Leibler's mean-field theory in order to obtain more reasonable results. Therefore, the model fit using eqs 8 to 14 qualitatively indicates that there is a significant decrease in micelle number density above the cmt.

Effect of Solvent Selectivity. The universality of these phenomena can be explored by inverting the solvent selectivity, i.e., to place the PS blocks in the micellar cores. SANS measurements for a $25 \%$ solution

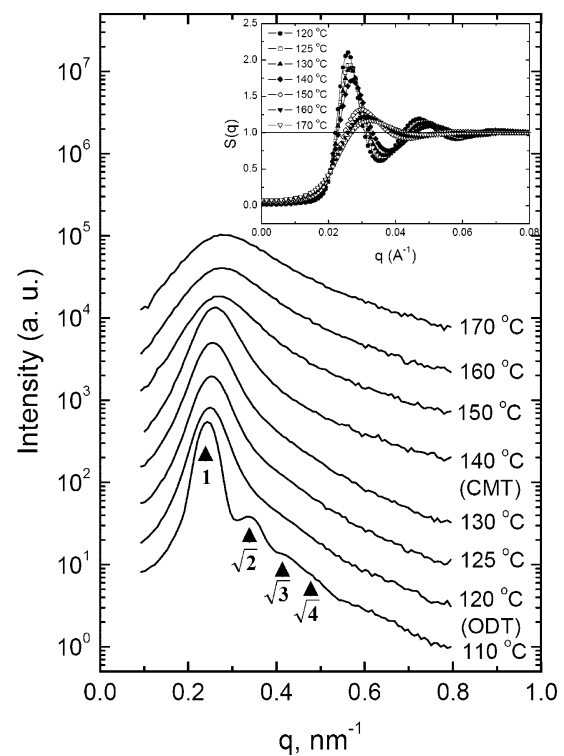

Figure 5. SANS profiles for a $25 \% d \mathrm{SI}(16-15)$ in $\mathrm{C} 14 / d \mathrm{C} 14$ $(90 / 10 \mathrm{vol} \%)$ with the increase in temperature. The filled triangles for the SANS profile at $115^{\circ} \mathrm{C}$ indicate the Bragg reflections corresponding to bcc phase. Each profile is shifted vertically by half a decade relative to the profile for immediately previous temperature for clarity. $T_{\mathrm{ODT}}$ is located at $120^{\circ} \mathrm{C}$ and an abrupt decrease in the peak intensity, which is related to the $\mathrm{cmt}$, was observed at around $140{ }^{\circ} \mathrm{C}$. The structure factors obtained from the GIFT model are also shown in the inset.

Table 4. Summary of GIFT Fitting Parameters for a $25 \%$ $d \mathrm{SI}(16-15)$ in $\mathrm{C} 14 / d \mathrm{C} 14(90 / 10$ vol \%)

\begin{tabular}{ccccc}
\hline$T,{ }^{\circ} \mathrm{C}$ & $R_{\mathrm{c}}, \AA$ & $R_{\mathrm{hs}}, \AA$ & $R_{\mathrm{hs}}-R_{\mathrm{c}}, \AA$ & $\phi_{\mathrm{hs}}$ \\
\hline 120 & 97.5 & 138.7 & 41.2 & 0.515 \\
122 & 96.2 & 137.1 & 40.9 & 0.508 \\
124 & 95.0 & 135.8 & 40.8 & 0.502 \\
126 & 94.0 & 134.7 & 40.7 & 0.497 \\
128 & 92.7 & 133.1 & 40.4 & 0.490 \\
130 & 91.3 & 131.7 & 40.4 & 0.483 \\
135 & 90.0 & 130.9 & 40.9 & 0.476 \\
140 & 89.3 & 128.8 & 39.5 & 0.466 \\
150 & 78.8 & 115.2 & 36.4 & 0.396 \\
160 & 73.3 & 109.4 & 36.1 & 0.355 \\
170 & 69.1 & 105.2 & 36.1 & 0.334
\end{tabular}

of $d \mathrm{SI}(16-15)$ in $\mathrm{C} 14 / d \mathrm{C} 14(90 / 10$ vol \%) solution were carried out, which also undergoes the bcc-to-disordered transition. As shown in Figure 5, the solution shows the ODT between 110 and $120{ }^{\circ} \mathrm{C}$ and a distinct $\mathrm{cmt}$ between 130 and $140{ }^{\circ} \mathrm{C}$. The data were analyzed by GIFT in exactly the same manner as for $\mathrm{S} d \mathrm{I}(15-14)$ in DEP/DBP. As shown in the inset of Figure 5, the first peak intensities of the structure factors at different temperatures above the ODT decrease considerably when undergoing the $\mathrm{cmt}$, and the oscillation of the structure factor eventually disappears upon further heating. The decrease in the $d \mathrm{PS}$ core size with the increase in temperature is again clearly represented as summarized in Table 4. In particular, we observed an abrupt decrease in $R_{c}$ around the cmt, which is similar to the behavior obtained with the $25 \% \mathrm{~S} d \mathrm{I}(15-14)$ in DEP/DBP. As shown in Table 4, the $\phi_{h s}$ gradually decreases throughout the temperature range covered in present study, and most notably, a significant decrease in $\phi_{h s}$ near the cmt is again detected. Similar features of $I_{\max }{ }^{-1}, \sigma_{q}{ }^{2}$, and $p(r)$ against temperature were obtained with the $25 \% d \mathrm{SI}(15-14)$ in $\mathrm{C} 14 / d \mathrm{C} 14$, as shown in Figure 6 . The experimentally determined $T_{\mathrm{ODT}}, T_{\mathrm{S}}$, and $T_{\mathrm{MF}}$ are about $121{ }^{\circ} \mathrm{C}, 140{ }^{\circ} \mathrm{C}$, and $152{ }^{\circ} \mathrm{C}$, 


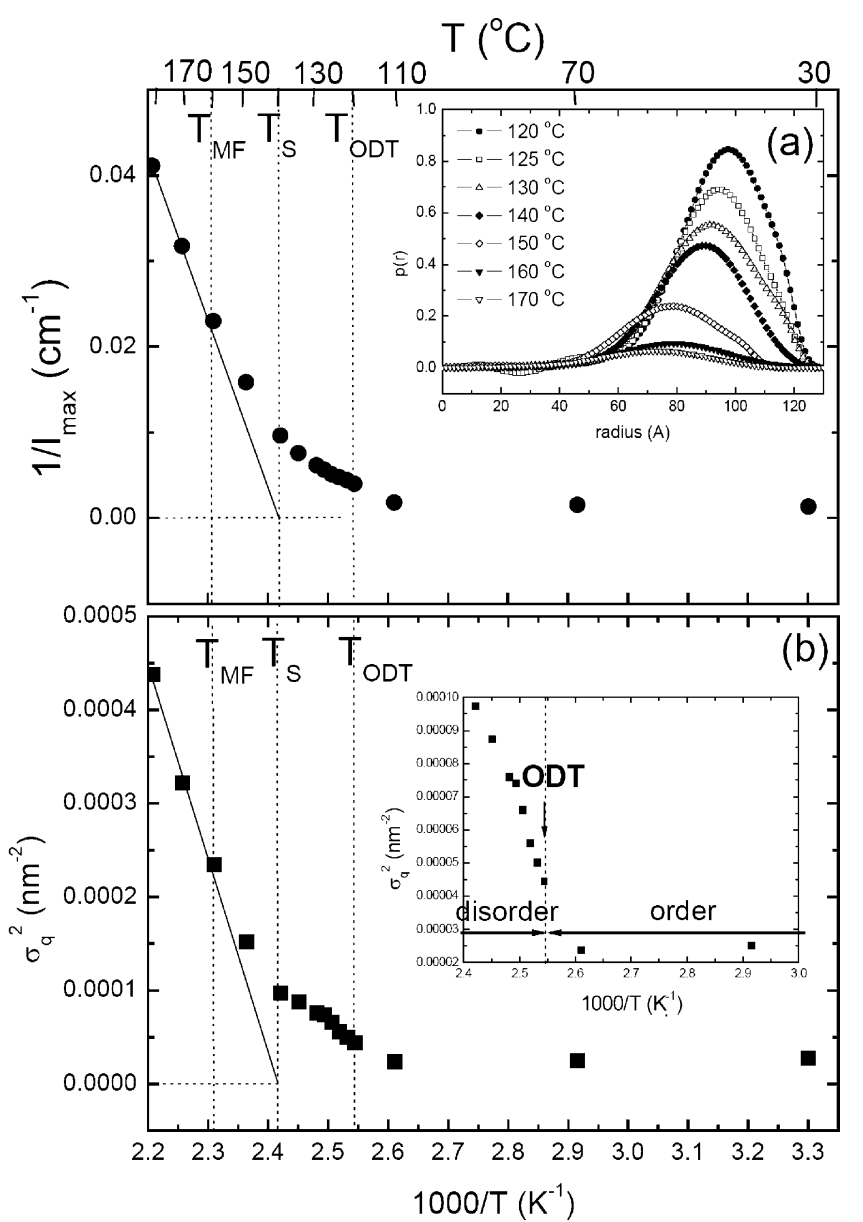

Figure 6. Temperature dependence of (a) $I_{\max }{ }^{-1}$ and (b) $\sigma_{q}{ }^{2}$ for a $25 \% d \mathrm{SI}(16-15)$ in $\mathrm{C} 14 / d \mathrm{C} 14(90 / 10 \mathrm{vol} \%)$ solution. The lines in parts $\mathrm{a}$ and $\mathrm{b}$ are fitted to the data as predicted from the mean-field theory. The inset in part a shows the pair distance distribution functions, $p(r)$, and the inset in part $b$ highlights the discontinuous change near the ODT. The meanfield temperature $\left(T_{\mathrm{MF}}\right)$, spinodal temperature $\left(T_{\mathrm{S}}\right)$, and orderdisorder transition temperature $\left(T_{\mathrm{ODT}}\right)$ are also located in the figure.

respectively. It is again interesting to note that the mean-field spinodal temperature $T_{\mathrm{S}}$ is close to the $\mathrm{cmt}$ as discussed in Figures 1 and 2. It is also worthwhile to note at this point that the temperature difference between the ODT and the cmt is about $20-30{ }^{\circ} \mathrm{C}$ for both solutions undergoing the bcc-to-disorder transition, independent of solvent. Detailed analysis of the SANS profiles in the disordered state above $T_{\mathrm{MF}}$ will be given later via mean-field theory.

ODT from Hex Lattices. SANS experiments were also performed for a $40 \% \mathrm{~S} d \mathrm{I}(15-14)$ in $\mathrm{DEP} / \mathrm{DBP}$ undergoing the hex-to-disorder transition. Figure 7 shows the corresponding SANS profiles (shifted vertically for clarity) upon heating. The typical UCODT-type phase behavior was observed. Below $78^{\circ} \mathrm{C}$, the higher order peaks at $\sqrt{3} q_{\max }$ and $\sqrt{4} q_{\max }$ peaks clearly indicate the hex microstructure, which disappear at a temperature between 78 and $80^{\circ} \mathrm{C}$. As a result, the $T_{\text {ODT }}$ is located between 78 and $80{ }^{\circ} \mathrm{C}$. Using the same criterion for the cmt established in Figure 1, we observe the onset of $\mathrm{cmt}$ at around $100{ }^{\circ} \mathrm{C}$. However, the extent of the decrease in the peak intensity near the $\mathrm{cmt}$ is not as distinct for the higher concentration. Upon further heating, the broad scattering maximum is consistent with a fully disordered block copolymer solution.

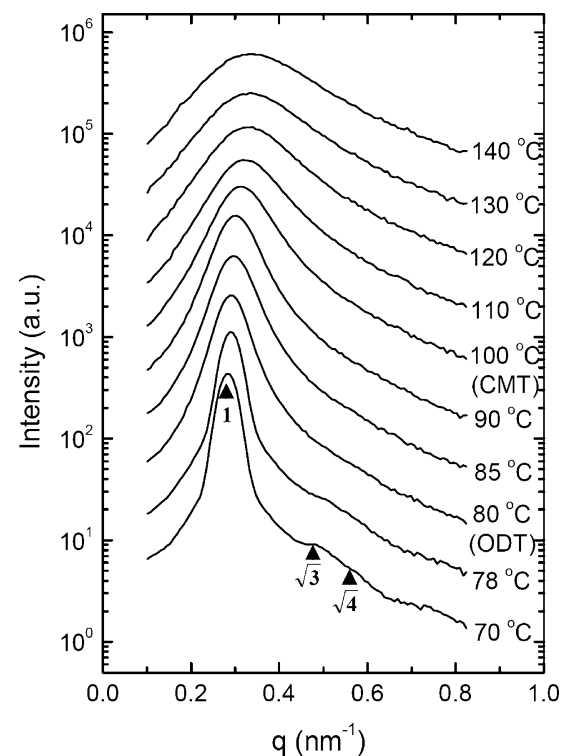

Figure 7. SANS profiles for a $40 \% \mathrm{~S} d \mathrm{I}(15-14)$ in DEP/DBP $(75 / 25 \mathrm{vol} \%)$ solution as a function of temperature. The filled triangles for the SANS profile at $70{ }^{\circ} \mathrm{C}$ indicate the Bragg reflections corresponding to the hexagonal cylinder phase. Each trace is shifted vertically by half a decade relative to the profile for immediately previous temperature for clarity. Both $T_{\text {ODT }}$ and $\mathrm{cmt}$ are located at 80 and $100{ }^{\circ} \mathrm{C}$, respectively, in the figure.

Different behavior of the disordered micelles by changing the polymer concentration can be shown by the plots of $I_{\max }{ }^{-1}, \sigma_{q}{ }^{2}$, and $D$ against $T^{-1}$, as presented in Figure 8 . The distinct $T_{\mathrm{ODT}}=80^{\circ} \mathrm{C}$ is located by the discontinuities in both $I_{\max }{ }^{-1}$ and $\sigma_{q}{ }^{2}$. From the $T^{-1}$ dependence of $D, T_{\mathrm{MF}} \approx 120^{\circ} \mathrm{C}$ was determined. In the same way as shown in Figure 2, the mean-field spinodal temperature, $T_{\mathrm{S}} \approx 100{ }^{\circ} \mathrm{C}$, which is related to the $\mathrm{cmt}$ as denoted in Figure 7, is determined. However, that the change of the slope of $D$ plotted against $T^{-1}$ near $T_{\mathrm{MF}}$ for the $40 \%$ solution is quite small compared with the visible change for the $25 \%$ solution. In fact, above the hex-to-disorder transition the cmt is harder to find compared with the bcc-to-disorder transition. We speculate that the difference arises because the cmt in the hex case is for a higher concentration solution, and the fully disordered fluid scattering (the "correlation hole" peak) is proportionally stronger; consequently, there is simply less difference between the disordered liquid and disordered micelle scattering. This interpretation is supported by previous work in melts, where the cmt was very hard to locate precisely. ${ }^{31}$

The GIFT analysis was also applied to obtain information on the micelle size and hard sphere volume fraction. The results are given in Table 5. Similarly, the PY average hard-sphere model, combined with an unconstrained form factor for a polydisperse system, also fits the data quite well and the fitting results are summarized in Table 6. The main features of the parameters obtained from the model fit are qualitatively similar to the case with bcc-to-disorder transition (i.e., $25 \%$ solutions). Figures $9 \mathrm{a}$ and $9 \mathrm{~b}$ show the pair distance distribution functions, $p(r)$, and the structure factors $S(q)$ at different temperatures, respectively. The decrease in the $d$ PI core size with almost constant effective corona thickness (ca. 3.1-3.7 $\mathrm{nm}$ ) and the steady decrease in $\phi_{h s}$ upon heating are again observed. A notable decrease in $R_{c}$ around $100{ }^{\circ} \mathrm{C}$, which is believed to be the cmt, is again present although it was 


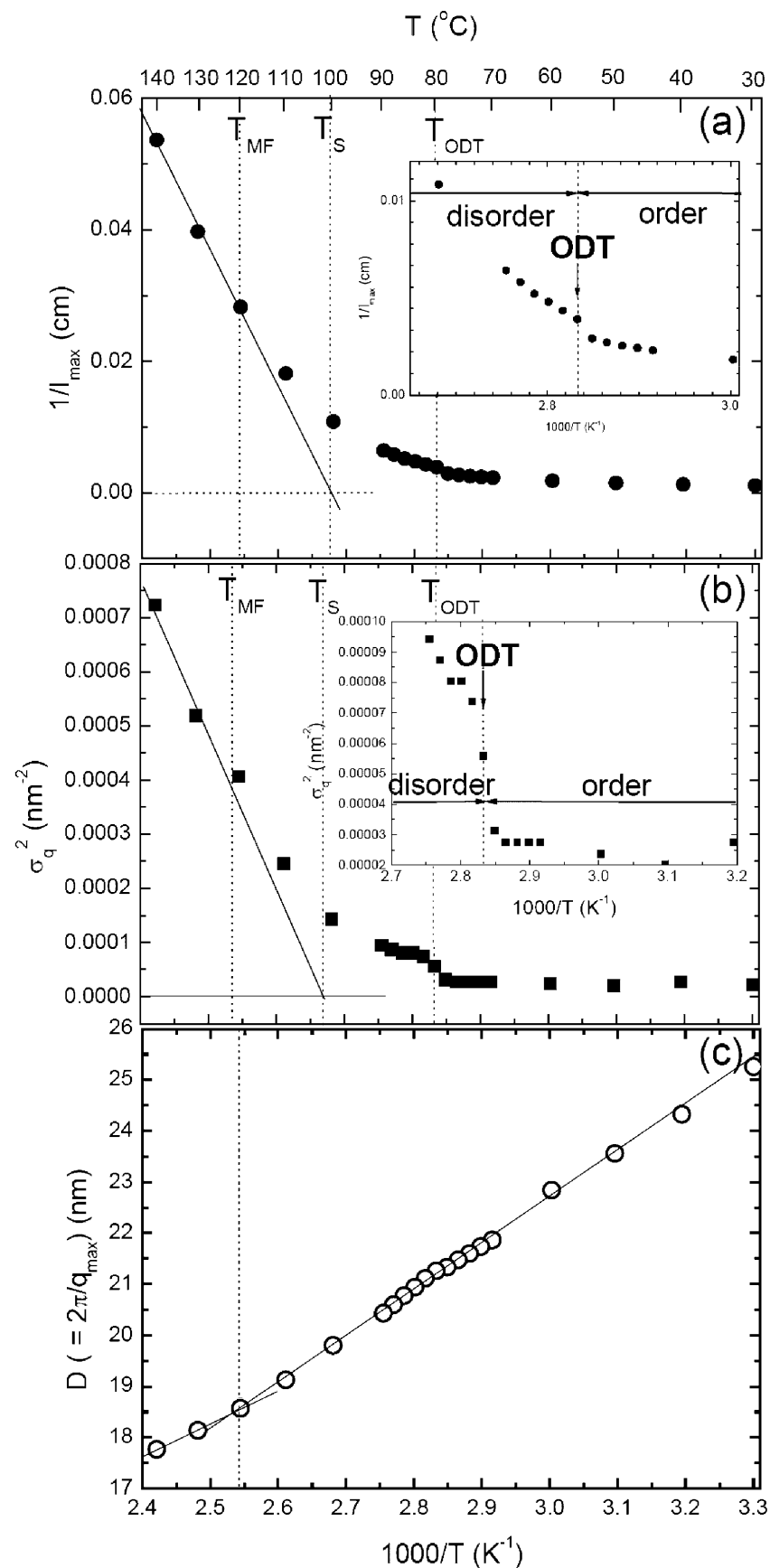

Figure 8. Temperature dependence of (a) $I_{\max }{ }^{-1}$, (b) $\sigma_{q}{ }^{2}$, and (c) $D$ for a $40 \% \mathrm{~S} d \mathrm{I}(15-14)$ in DEP/DBP $(75 / 25$ vol \%) solution. The lines in parts $\mathrm{a}$ and $\mathrm{b}$ are fitted to the data as predicted from the mean-field theory. Insets in parts $a$ and $b$ highlight the discontinuous change in both quantities near the ODT and the positions of $T_{\mathrm{MF}}, T_{\mathrm{S}}$, and $T_{\mathrm{ODT}}$ are indicated in the figure.

Table 5. Summary of GIFT Fitting Parameters for a $40 \%$ SdI $(15-14)$ in DEP/DBP $(75 / 25$ vol \%)

\begin{tabular}{rrrcc}
\hline$T,{ }^{\circ} \mathrm{C}$ & $R_{\mathrm{c}}, \AA$ & $R_{\mathrm{hs}}, \AA$ & $R_{\mathrm{hs}}-R_{\mathrm{c}}, \AA$ & $\phi_{\mathrm{hs}}$ \\
\hline 80 & 81.8 & 118.5 & 36.7 & 0.525 \\
85 & 79.5 & 114.4 & 34.9 & 0.492 \\
90 & 77.7 & 111.6 & 33.9 & 0.479 \\
100 & 70.9 & 104.1 & 33.2 & 0.445 \\
110 & 62.7 & 98.0 & 35.3 & 0.392 \\
120 & 60.5 & 92.7 & 32.7 & 0.383 \\
130 & 57.7 & 89.3 & 31.6 & 0.351 \\
140 & 55.9 & 86.4 & 30.5 & 0.318
\end{tabular}

harder to locate the cmt from the scattering profiles given in Figure 7. However, it should be mentioned here that the extent of decrease in $R_{c}$ and $\phi_{h s}$ for the hex-to-
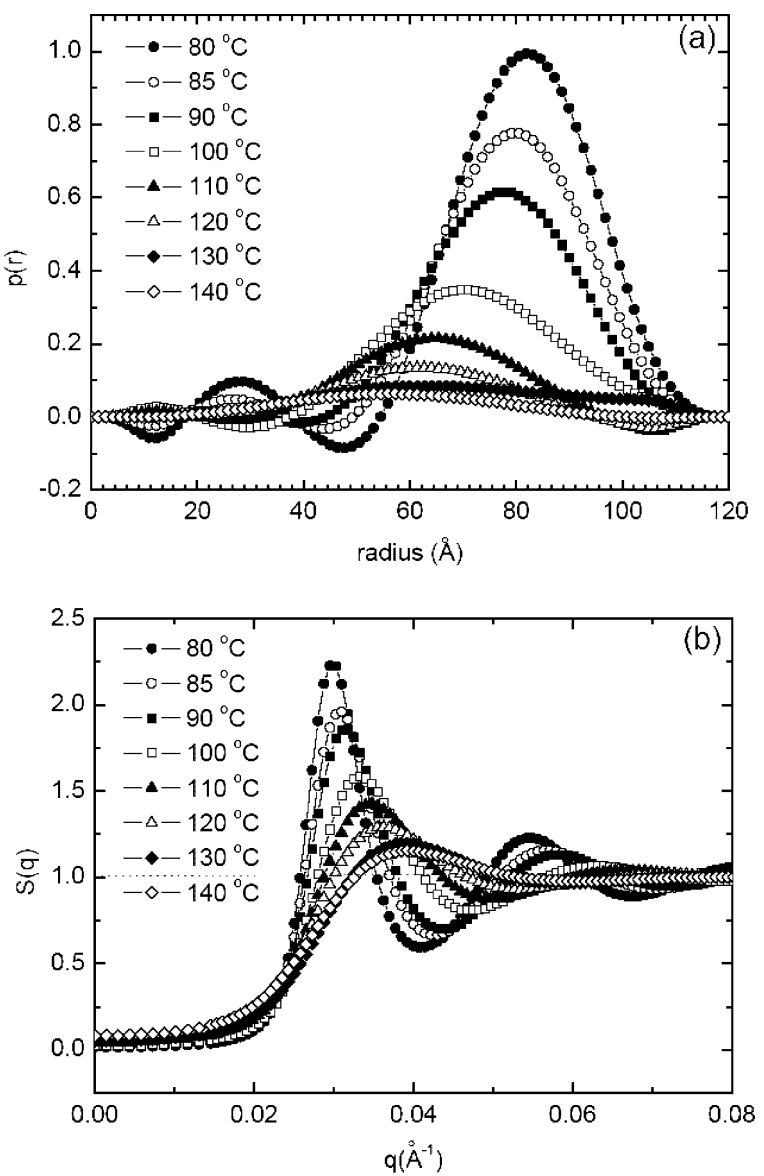

Figure 9. Representative (a) pair distance distribution functions, $p(r)$ and (b) structure factors $S(q)$ obtained from the GIFT at indicated temperatures for a $40 \% \mathrm{~S} d \mathrm{I}(15-14)$ in DEP/ DBP $(75 / 25$ vol \%) solution.

Table 6. Summary of the Direct Spherical Model Fits for a $40 \% \mathrm{SdI}(15-14)$ in DEP/DBP $(75 / 25$ vol \%)

\begin{tabular}{rrrccccc}
\hline$T,{ }^{\circ} \mathrm{C}$ & $R_{\mathrm{c}}, \AA$ & $R_{\mathrm{hs}}, \AA$ & $R_{\mathrm{hs}}-R_{\mathrm{c}}, \AA$ & $N_{\text {agg }}$ & $\phi_{\text {core, sol }}$ & $\sigma_{\mathrm{c}}$ & $\phi_{\mathrm{hs}}$ \\
\hline 80 & 79.3 & 120.5 & 41.2 & 56 & 0.366 & 7.7 & 0.520 \\
85 & 77.5 & 117.2 & 39.7 & 51 & 0.384 & 7.8 & 0.495 \\
90 & 74.8 & 112.0 & 37.2 & 45 & 0.395 & 7.6 & 0.473 \\
100 & 68.5 & 106.3 & 37.8 & 32 & 0.427 & 9.4 & 0.438 \\
110 & 60.7 & 100.1 & 39.4 & 21 & 0.473 & 8.9 & 0.383 \\
120 & 60.4 & 95.0 & 34.6 & 18 & 0.549 & 11.4 & 0.367 \\
130 & 60.5 & 90.8 & 30.3 & 17 & 0.568 & 13.9 & 0.339 \\
140 & 55.9 & 88.4 & 32.5 & 12 & 0.609 & 14.1 & 0.308
\end{tabular}

disorder transition is approximately half what it was for the bcc-to-disorder transition. Above the cmt, the oscillations in the correlation functions gradually disappear and eventually level off to unity, implying the low intermicellar correlation due to the formation of dominant free chains.

The direct model fitting was carried out with a spherical form factor (eq 9) along with the PY structure factor (eq 11) with the core size polydispersity (eq 10) and the instrumental smearing effects (eq 13) taken into account. One might imagine that the hex phase could disorder into wormlike micelles rather than spheres. Accordingly, we also tried the direct model fitting with an ellipsoidal form factor, considering the ellipticity of micelles, $\epsilon$, as follows: ${ }^{53}$

$$
\begin{gathered}
P_{\text {core }}(q)=\int_{0}^{\pi / 2} P^{2}[q, r(R, \epsilon, \alpha)] \sin \alpha \mathrm{d} \alpha \\
r(R, \epsilon, \alpha)=R\left(\sin ^{2} \alpha+\epsilon^{2} \cos \alpha\right)^{1 / 2}
\end{gathered}
$$




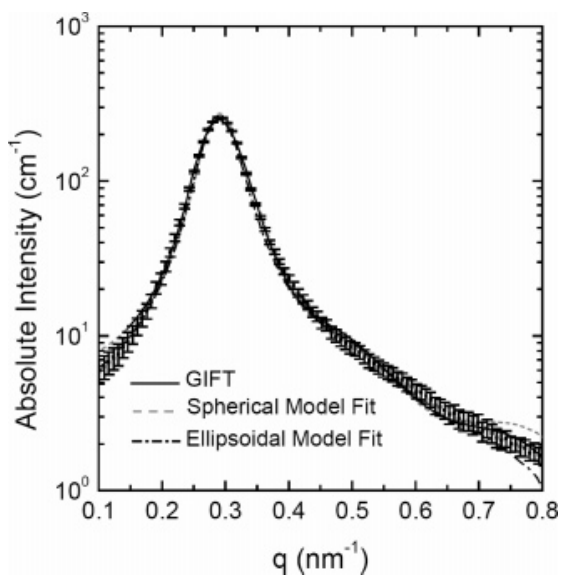

Figure 10. SANS scattering profile for a $40 \% \mathrm{~S} d \mathrm{I}(15-14)$ in DEP/DBP $\left(75 / 25\right.$ vol \%) solution at $80^{\circ} \mathrm{C}$ filled with the GIFT and the PY effective hard-sphere model with spherical and ellipsoidal form factors.

However, we were unable to find a better fit than with the spherical form factor. The best fit with the ellipsoidal form factor is obtained with $\epsilon=1$.3. Figure 10 shows representative fitting results for the SANS profile taken at $80{ }^{\circ} \mathrm{C}$. Both GIFT and direct model fitting yields good agreement with the experimental data. The aggregation number of the micelles is again estimated by allowing the presence of solvent in the micellar core (eq 14), and the calculated values are also summarized in Table 6. With increasing temperature, the aggregation number steadily decreases with the increased solvent swelling of the core. At the cmt, the obtained aggregation number is about 30 and the volume fraction of solvent is as high as $40 \%$, implying denser micellar cores compared with the $25 \%$ solution. Upon further heating, the aggregation number decreases close to 10 and more than $50 \%$ solvent exists in the micelle core indicating a significant fraction of free chains in solution with increasing temperature. Again, the micelles were found to dissociate into free chains at approximately 20 ${ }^{\circ} \mathrm{C}$ higher than $T_{\mathrm{ODT}}$ and the mean-field fits above $T_{\mathrm{MF}}$ will be described below.

Crossover from Disordered Micelles to MeanField Regime above the cmt. $I(q)$ from a block copolymer solution in the disordered state within the mean-field regime is described by ${ }^{54}$

$$
I(q) \sim\left[S(q) / W(q)-2 \chi_{e f f}\right]^{-1}
$$

where $S(q)$ is the sum of all elements and $W(q)$ is the determinant of the matrix:

$$
\begin{gathered}
S(q)=S_{\mathrm{AA}}(q)+S_{\mathrm{BB}}(q)+2 S_{\mathrm{AB}}(q)=N g(1) \\
W(q)=S_{\mathrm{AA}}(q) S_{\mathrm{BB}}(q)-S_{\mathrm{AB}}^{2}(q)
\end{gathered}
$$

with

$$
\begin{gathered}
S_{\mathrm{AA}}(q)=N g(f) \\
S_{\mathrm{BB}}(q)=N g(1-f) \\
S_{\mathrm{AB}}(q)=N / 2[g(1)-g(f)-g(1-f)] \\
g(f)=\left(2 / x^{2}\right)[f x+\exp (-f x)-1] \\
x=q^{2} R_{g}^{2}
\end{gathered}
$$

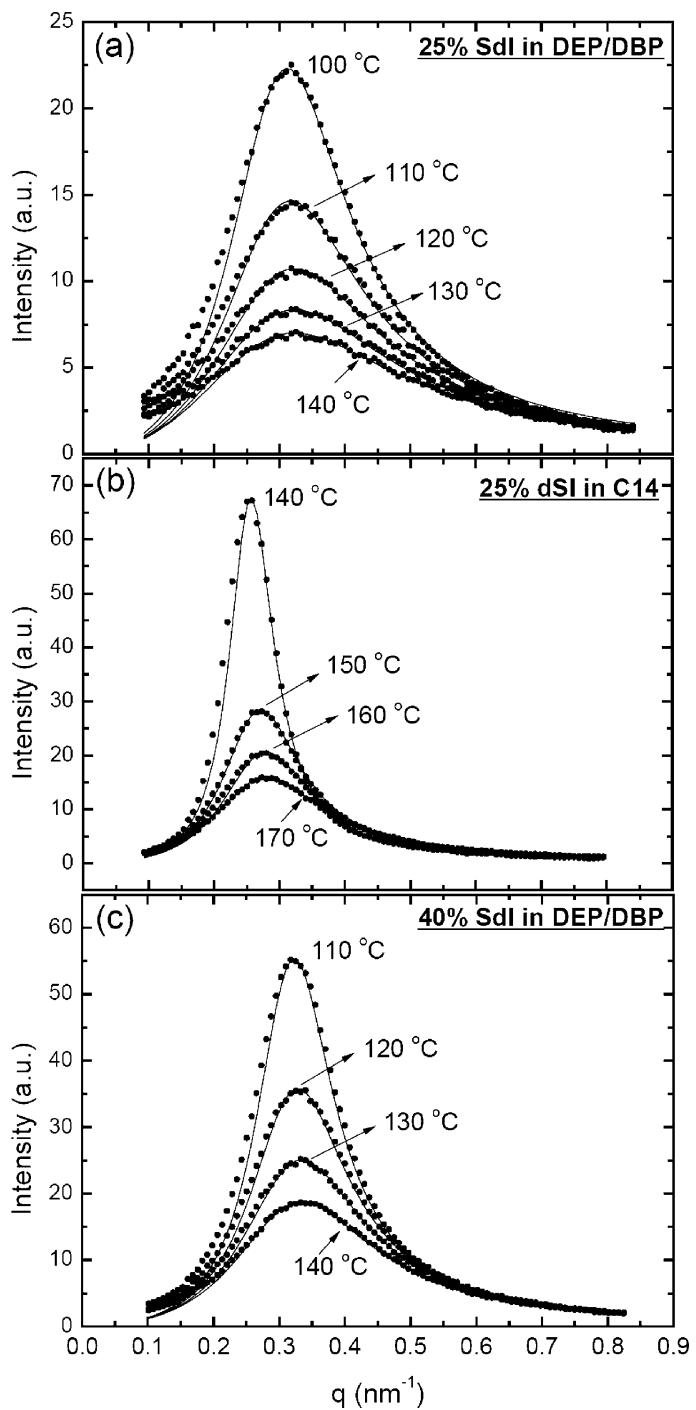

Figure 11. SANS profiles at different temperatures above the cmt for (a) a $25 \% \mathrm{~S} d \mathrm{I}(15-14)$ in DEP/DBP (75/25 vol \%), (b) a $25 \% d \mathrm{SI}(16-15)$ in $\mathrm{C} 14 / d \mathrm{C} 14(90 / 10 \mathrm{vol} \%)$, and (c) a $40 \%$ $\mathrm{S} d \mathrm{I}(15-14)$ in DEP/DBP (75/25 vol \%). The solid lines show the scattering profiles predicted from the Leibler-Landau mean-field theory in disordered state.

Since $I(q)$ defined by eqs $17-24$ is only applied to monodisperse systems, we extended the Leibler-Landau-type mean-field theory ${ }^{1}$ for the disordered regime accounting for the effect of polydispersity in the molecular weight and the solvent effect, which have previously been demonstrated to be important in some cases..$^{55}$ Accordingly, $g(f)$ is replaced by

$$
N g(f)=2 N_{\mathrm{n}} / x^{2}\left\{f x-1+[k /(k+f x)]^{k}\right\}
$$

with

$$
\begin{gathered}
N_{\mathrm{n}}=\left(\rho_{P S} \rho_{\mathrm{PI}}\right)^{1 / 2}\left(N_{n, P S} / \rho_{\mathrm{PS}}+N_{n, P I} / \rho_{\mathrm{PI}}\right) \\
N_{\mathrm{w}} / N_{\mathrm{n}}=(k+1) / k
\end{gathered}
$$

where $\rho_{\text {PS }}$ and $\rho_{\text {PI }}$ are the segmental densities of PS and PI: $\rho_{\mathrm{PS}}=1.01 \times 10^{-2} \mathrm{~mol} / \mathrm{m}^{3} ; \rho_{\mathrm{PI}}=1.34 \times 10^{-2} \mathrm{~mol} / \mathrm{m}^{3}$ (for 1,4-addition). ${ }^{22}$ In our system, the polydispersity index is found to be 1.04 for both $\mathrm{S} d \mathrm{I}(15-14)$ and $d \mathrm{SI}$ $(16-15)$ with $k \approx 25$.

Figure 11 shows the scattering profiles from $25 \% \mathrm{~S} d \mathrm{I}$ in $\mathrm{DEP} / \mathrm{DBP}, 25 \% d \mathrm{SI}$ in $\mathrm{C} 14 / d \mathrm{C} 14$, and $40 \% \mathrm{~S} d \mathrm{I}$ in 
Table 7. Summary of the Leibler-Landau Fit Results for $25 \%$ SdI $(15-14)$ in DEP/DBP (75/25 Vol \%), 25\%

$d \mathrm{SI}(16-15)$ in $\mathrm{C} 14 / d \mathrm{C} 14(90 / 10$ vol \%), and $40 \% \mathrm{~S} d \mathrm{I}(15-14)$ in DEP/DBP (75/25 vol \%)

\begin{tabular}{cccc}
\hline & \multicolumn{3}{c}{$\mathrm{R}_{\mathrm{g}}, \AA$} \\
$T,{ }^{\circ} \mathrm{C}$ & $\begin{array}{c}25 \% \mathrm{~S} d \mathrm{I}(15-14) \\
\text { in DEP/DBP }\end{array}$ & $\begin{array}{c}25 \% d \mathrm{SI}(16-15) \\
\text { in C14/dC14 }\end{array}$ & $\begin{array}{c}40 \% \mathrm{~S} d \mathrm{I}(15-14) \\
\text { in DEP/DBP }\end{array}$ \\
\hline 100 & 60 & & 58 \\
110 & 58 & & 57 \\
120 & 58 & & 56 \\
130 & 58 & 72 & 56 \\
140 & 58 & 69 & \\
150 & & 67 & \\
160 & & 67 & \\
170 & & &
\end{tabular}

DEP/DBP solutions in the disordered state above the cmt as a function of temperature. The solid lines are obtained by the fitting with eqs $17-27$, and the results are summarized in Table 7. All the solutions given in Figure 11 show scattering profiles with single peaks. According to the mean-field theory the scattering vector at the intensity maximum $I_{\max }, q_{\max }$, is independent of temperature and the $I_{\max }$ decreases as temperature $T$ is increased. The $q_{\max }$ is also related to the correlation holes reflecting the dominant mode of concentration fluctuations on the scale of $R_{\mathrm{g}}$. It is particularly interesting to note that three solutions in Figure 11 show the temperature independence of $q_{\max }$ at high temperature, which is in agreement with the Leibler-Landau theory, and as the temperature is lowered there exists an onset temperature at which the $q_{\max }$ deviates from the $q_{\max }$ at the high temperature (also see Table 7). The small shift in $q_{\max }$ reflects the incipient point of the nonmean-field effect. ${ }^{3}$ Accordingly, the $T_{\mathrm{MF}}$ can be accurately determined by this criterion and the $T_{\mathrm{MF}}$ values thus determined are between 100 and $110{ }^{\circ} \mathrm{C}$ for a $25 \% \mathrm{~S} d \mathrm{I}$ in $\mathrm{DEP} / \mathrm{DBP}$, between 150 and $160^{\circ} \mathrm{C}$ for a $25 \% d \mathrm{SI}$ in $\mathrm{C} 14 / d \mathrm{C} 14$, and between 120 and $130{ }^{\circ} \mathrm{C}$ for a $40 \%$ solution of $\mathrm{S} d \mathrm{I}$ in $\mathrm{DEP} / \mathrm{DBP}$, which are in excellent agreement with the values obtained by the $I_{\max }{ }^{-1}$ vs $T^{-1}$ plots shown in Figures 2,6 , and 8 . The comparison between the experimental data and the theoretical predictions also shows some deviations at low $q\left(<q_{\max }\right)$, and this discrepancy at low $q$ tends to increase with increasing temperature. We tentatively attribute this to an increased contribution of concentration fluctuations between solvent and copolymer chains. ${ }^{22}$

On the basis of these experimental results, a schematic illustrating the regions of the micelles in longrange order, the disordered micelles, and the dominant free chains along with associated transitions is given in Figure 12. For $\mathrm{S} d \mathrm{I}(15-14)$ in DEP/DBP, the solution with polymer volume fraction $\phi=0.25$ adopts the bcc structure while for $\phi=0.40$, the hex phase is observed. The long-range order for both bcc and hex phases is maintained up to the $T_{\mathrm{ODT}}$, at which the long-range lattice order is lost. At approximately $20-30{ }^{\circ} \mathrm{C}$ higher than $T_{\mathrm{ODT}}$, the disordered micelles were found to dissociate into free chains, which is defined as the cmt in present study. As a result, upon heating from a lowtemperature ordered state, the balance between the chains in micelles and the free chains is shifted toward the free chains, eventually disrupting all the micelles at around $T_{\mathrm{MF}}$. The observed phase sequence is similar to that in bcc-forming block copolymer melts: ${ }^{26,30-33}$ one thermodynamic phase transition, the ODT, and one phenomenological transition, the cmt, which lies within the disordered phase.

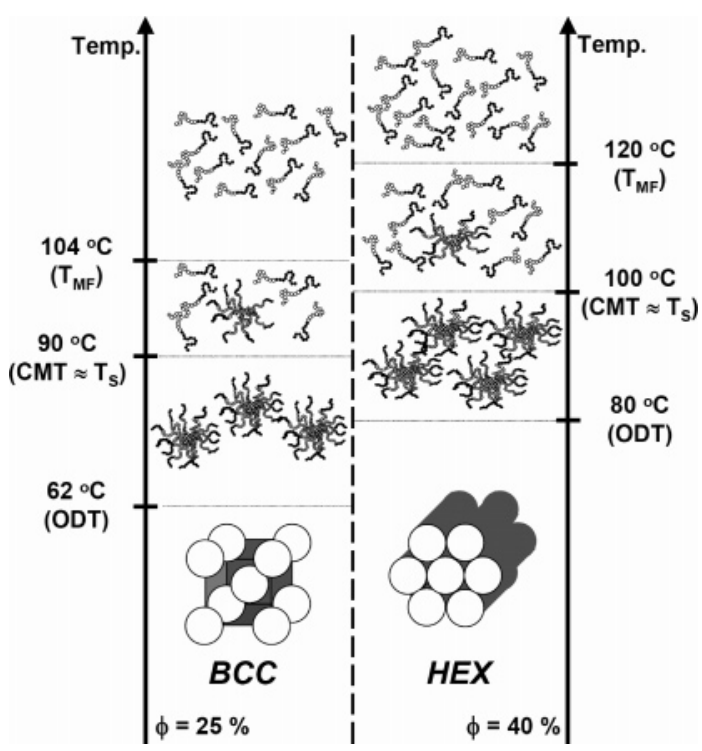

Figure 12. Phase behavior for $25 \% \mathrm{~S} d \mathrm{I}(15-14)$ in $\mathrm{DEP} / \mathrm{DBP}$ $(75 / 25$ vol \%) and $40 \% \mathrm{~S} d \mathrm{I}(15-14)$ in DEP/DBP $(75 / 25$ vol \%). Bcc and HEX denote the body-centered cubic and the hexagonal cylinder, respectively, and ODT, cmt, $T_{\mathrm{S}}$, and $T_{\mathrm{MF}}$ for the two solutions are shown. A schematic illustrating the regions of the micelles in long-range order, the disordered micelles along with associated transitions is also given.

\section{Conclusions}

1. We have examined the solution behavior just above $T_{\mathrm{ODT}}$ by SANS with symmetric PS- $d \mathrm{PI}$ and $d \mathrm{PS}-\mathrm{PI}$ diblock copolymers in selective solvents. The modelindependent GIFT of the experimental data and the direct model fitting with appropriate form factors and structure factors were employed to analyze the SANS data to make a close investigation of the solution behavior in the disordered micelle regime.

2. The micelles were found to break up into free chains at the cmt, which is experimentally determined by an abrupt decrease in the size, aggregation number, and volume fraction of micelles and is close to $T_{\mathrm{S}}$. Notably, regardless of different ordered microstructures prior to the disordered state, i.e., bcc and hex, the cmt is approximately $20-30{ }^{\circ} \mathrm{C}$ higher than $T_{\text {ODT }}$.

3 . It is interesting to note that for solutions undergoing the bcc-to-disorder transition, a clear crossover behavior of the domain spacing $(D)$ upon heating across the $T_{\mathrm{MF}}$ was observed. However, the slope of $D$ vs $T^{-1}$ does not change around $T_{\mathrm{MF}}$ for the hex-to-disorder transition. In addition, for the hex-to-disorder transition, the cmt is harder to locate compared with bcc-todisorder transition. Interestingly, even in this case the disordered micelles were approximately spherical.

4. Two transitions, one between ordered phase and disordered micelles (ODT) and the other between disordered micelles and disorder $(\mathrm{cmt})$ were identified and consequently, three clear regimes were discussed: ordered-ODT-disordered micelles-cmt-mean field regime.

Acknowledgment. This work was supported primarily by the MRSEC program of the National Science Foundation under Award DMR-0212302. We are grateful to the financial support from the National Research Laboratory Fund (Grant M1-0104-00-0191) from the Ministry of Science and Technology of Korea and the Brain Korea 21 Program endorsed by the Ministry of Education of Korea. Further support for this research 
was provided by the National Institute of Standards and Technology, U.S. Department of Commerce, through the neutron research facilities.

\section{References and Notes}

(1) Leibler, L. Macromolecules 1980, 13, 1602

(2) Helfand, E.; Wasserman, Z. R. Developments in Block Copolymers; Goodman, I., Ed.; Applied Science: New York, 1985; Chapter 4.

(3) Fredrickson, G. H.; Helfand, E. J. Chem. Phys. 1987, 87, 697.

(4) Brown, R. A.; Masters, A. J.; Price, C.; Yuan, X. F. In Comprehensive Polymer Science; Booth, C.; Price, C., Eds.; Pergamon Press: Oxford, U.K., 1989; Vol. 2.

(5) Tuzar, Z.; Kratochvil, P. In Surface and Colloid Science; Matijevic, E., Ed.; Plenum Press: New York, 1993; Vol. 15.

(6) Mortensen, K.; Pedersen, J. S. Macromolecules 1993, 26, 805

(7) Mayes, A. M.; Barker, J. G.; Russell, T. P. J. Chem. Phys. 1994, 101, 5213.

(8) Lodge, T. P.; Xu, X.; Ryu, C. Y.; Hamley, I. W.; Fairclough, J. P. A.; Ryan, A. J.; Pedersen, J. S. Macromolecules 1996 29,5955

(9) Adams, J. L.; Quiram, D. J.; Graessley, W. W.; Register, R. A.: Marchand, G. R. Macromolecules 1996, 29, 2929.

(10) Schwab, M.; Stühn, B. Phys. Rev. Lett. 1996, 76, 924.

(11) Maurer, W. W.; Bates, F. S.; Lodge, T. P.; Almdal, K. Mortensen, K.; Fredrickson, G. H. J. Chem. Phys. 1998, 108, 2989.

(12) Bates, F. S.; Rosedale, J. H.; Fredrickson, G. H.; Glinka, C. J. Phys. Rev. Lett. 1988, 61, 2229.

(13) Bates, F. S.; Rosedale, J. H.; Fredrickson, G. H. J. Chem. Phys. 1990, 92, 6255

(14) Rosedale, J. H.; Bates, F. S. Macromolecules 1990, 23, 2329

(15) Almdal, K.; Bates, F. S.; Mortensen, K. J. Chem. Phys. 1992 96,9122

(16) Rosedale, J. H.; Bates, F. S.; Almdal, K.; Mortensen, K. Wignall, G. D. Macromolecules 1995, 28, 1429.

(17) Frielinghaus, H.; Schwahn, D.; Mortensen, K.; Almdal, K. Springer, T. Macromolecules 1996, 29, 3263.

(18) Holmqvist, P.; Pispas, S.; Hadjichristidis, N.; Fytas, G.; Sigel, R. Macromolecules 2003, 36, 830

(19) Guenza, M.; Schweizer, K. S. J. Chem. Phys. 1997, 106, 7391

(20) Guenza, M.; Schweizer, K. S. Macromolecules 1997, 30, 4205.

(21) Almdal, K.; Mortensen, K.; Ryan, A. J.; Bates, F. S. Macromolecules 1996, $29,5940$.

(22) Mori, K.; Okawara, A.; Hashimoto, T. J. Chem. Phys. 1996 104,7765 .

(23) Adams, J. L.; Graessley, W. W.; Register, R. A. Macromolecules 1994, 27, 6026.

(24) Zhao, J.; Majumdar, B.; Schulz, M. F.; Bates, F. S.; Almdal, K.; Mortensen, K.; Hajduk, D. A.; Gruner, S. M. Macromolecules 1996, 29, 1204

(25) Lee, S.-H.; Char, K.; Kim, G. Macromolecules 2000, 33, 7072.

(26) Dormidontova, E. E.; Lodge, T. P. Macromolecules 2001, 34, 9143.

(27) Sota, N.; Sakamoto, N.; Saijo, K.; Hashimoto, T. Macromolecules 2003, 36, 4534.
(28) Sakamoto, N.; Hashimoto, T.; Han, C. D.; Kim, D.; Vaidya, N. Y. Macromolecules 1997, 30, 1621.

(29) Sakamoto, N.; Hashimoto, T.; Han, C. D.; Kim, D.; Vaidya, N. Y. Macromolecules 1997, 30, 5321.

(30) Han, C. D.; Vaidya, N. Y.; Kim, D.; Shin, G.; Yamaguchi, D.; Hashimoto, T. Macromolecules 2000, 33, 3767.

(31) Wang, X.; Dormidontova, E. E.; Lodge, T. P. Macromolecules 2002, 35, 9687

(32) Bodycomb, J.; Yamaguchi, D.; Hashimoto, T. Macromolecules $\mathbf{2 0 0 0}, 33,5187$

(33) Wang, J. F.; Wang, Z.-G.; Yang, Y. L. Macromolecules, in press.

(34) Semenov, A. N. Macromolecules 1989, 22, 2849.

(35) Matsen, M. W.; Bates, F. S. Macromolecules 1996, 29, 1091.

(36) Matsen, M. W.; Bates, F. S. J. Chem. Phys. 1997, 106, 2436.

(37) Shibayama, M.; Hashimoto, T.; Kawai, H. Macromolecules 1983, 16, 16.

(38) Hashimoto, T.; Shibayama, M.; Kawai, H. Macromolecules 1983, 16,361

(39) Hamley, I. W.; Fairclough, J. P. A.; Ryan, A. J.; Ryu, C. Y.; Lodge, T. P.; Gleeson, A. J.; Pedersen, J. S. Macromolecules 1998, 31,1188

(40) Hanley, K. J.; Lodge, T. P.; Huang, C.-I. Macromolecules 2000, 33, 5918.

(41) Lodge, T. P.; Hanley, K. J.; Pudil, B. Macromolecules 2002, $35,4707$.

(42) Bang, J.; Viswanathan, K.; Lodge, T. P.; Park, M. J.; Char, K. J. Chem. Phys. 2004, 121, 11489.

(43) Jackson, C.; Nillson, L.; Wyatt, P. J. J. Appl. Polym. Sci. 1991, $42,217$.

(44) Huglin, M. B. Light Scattering from Polymer Solution; Academic: New York, 1972.

(45) Park, M. J.; Bang, J.; Harada, T.; Lodge, T. P.; Char, K. Macromolecules 2004, 37, 9064.

(46) Brunner-Popela, J.; Glatter, O. J. Appl. Crystallogr. 1997, $30,431$.

(47) Hansen, J. P.; McDonald, I. R. In The Theory of Simple Liquids; Academic: London, 1990.

(48) Brunner-Popela, J.; Mittelbach, R.; Strey, R.; Schubert, K.V.; Kaler, E. W.; Glatter, O. J. Chem. Phys. 1999, 21, 10623

(49) Weyerich, B.; Brunner-Popela, J.; Glatter, O. J. Appl. Crystallogr. 1999, 32, 197

(50) Pedersen, J. S.; Gerstenberg, M. C. Macromolecules 1996, 29 , 1363.

(51) Pedersen, J. S. J. Chem. Phys. 2001, 114, 2839.

(52) Pedersen, J. S.; Posselt, D.; Mortensen, K. J. Appl. Crystallogr. 1990, 23, 321 .

(53) Feigin, L. A.; Svergun, D. I. In Structure Analysis by SmallAngle X-ray and Neutron Scattering; Plenum Press: New York, 1987.

(54) Benmouna, M.; Wu, W.; Mozer, B.; Bauer, B.; Lapp, A. Macromolecules 1985, 18, 986.

(55) Sakurai, S.; Mori, K.; Okawara, A.; Kimishima, K.; Hashimoto, T. Macromolecules 1992, 25, 2679.

MA0477044 